\title{
SED, metallicity and age of halo globular clusters in M 33
}

\author{
J. Ma, X. Zhou, J. Chen, H. Wu, Z. Jiang, S. Xue, and J. Zhu \\ National Astronomical Observatories, Chinese Academy of Sciences, Beijing, 100012, PR China \\ Received 29 May 2001 / Accepted 23 January 2002

\begin{abstract}
In this paper we study the properties of ten halo globular clusters in the nearby spiral galaxy M 33 . CCD images of M 33 were obtained as a part of the BATC Colour Survey of the sky in 13 intermediate-band filters from 3800 to $10000 \AA$. By aperture photometry, we obtain the spectral energy distributions (SEDs) of these globular clusters. We estimate the ages of our sample clusters by comparing the photometry of each object with theoretical stellar population synthesis models for different values of metallicity. Our results suggest that eight of the ten sample halo globular clusters have "intermediate" ages, i.e. between 1 and 8 Gyrs.
\end{abstract}

Key words. galaxies: photometry - galaxies: cluster: individual - galaxies: evolution

\section{Introduction}

Globular clusters are roughly spherical agglomerations of stars. It is generally believed that they are among the first objects to be formed in a galaxy. Their ages provide us with information on the early formative stages of the parent galaxy. The study of these systems has played a key role in the development of our understanding of the parent galaxy. For example, they can be utilized to provide a lower limit to the age of the parent galaxy provided their ages can be ascertained, and to study the properties of the parent galaxy soon after its formation.

In addition to the Milky Way, a number of other Local Group galaxies have been presented to contain globular clusters: for example, the Sagittarius dwarf spheroidal galaxy, the Large and Small Magellanic Clouds, M 31, and M33. Schommer et al. (1991) estimated the number of the total "true" globular cluster population of M 33 to be only $\sim 20$. Sarajedini et al. (1998) selected ten halo globular clusters from Schommer et al. (1991) by inspecting the difference between the cluster velocity and the disk velocity as a function of the integrated cluster colour, and constructed colour-magnitude diagrams to estimate the cluster metallicity using the shape and colour of the red giant branch. Under the assumption that cluster age is the global second parameter, Sarajedini et al. (1998) presented that the average age of halo globular clusters in M 33 appears to be a few Gyrs younger than halo clusters in the Milky Way.

In this paper, we present the SEDs of ten halo globular clusters of M 33 from Sarajedini et al. (1998), and estimate the metallicities and ages of these clusters by using

Send offprint requests to: J. Ma,

e-mail: majun@vega.bac.pku.edu.cn the theoretical evolutionary population synthesis methods (Bruzual \& Charlot 1996).

The outline of the paper is as follows. Details of observations and data reduction are given in Sect. 2. In Sect. 3, we provide a brief description of the stellar population synthesis models of Bruzual \& Charlot (1996). The metallicities and ages are estimated in Sect. 4. The summary is presented in Sect. 5.

\section{Sample of star clusters, observations and data reduction}

\subsection{Sample of star clusters}

The sample of halo globular clusters in this paper is from Sarajedini et al. (1998), who estimated their metallicities using the shape and colour of the red giant branch. The RAs and Decs of these clusters are from Christian \& Schommer (1982), who detected more than 250 nonstellar objects (including the sample halo globular clusters of this paper) using $14 \times 14$ inch $^{2}$ unfiltered, unbaked, IIa-O focus plate exposed for 150 minutes with the Kitt Peak $4 \mathrm{~m}$ Richey-Chrétien (R-C) direct camera. Because of distortion in the $4 \mathrm{~m}$ R-C focal plane, Christian \& Schommer (1982) presented the coordinates of the sample clusters to be good only to $\sim 20^{\prime \prime}$. We corrected the coordinates of our sample clusters using the HST Guide Star Catalog, and list them in Table 3.

\section{2. $C C D$ image observation}

The large field multi-colour observations of the spiral galaxy M33 were obtained in the BATC photometric system. The telescope used is the $60 / 90 \mathrm{~cm} \mathrm{f} / 3$ 


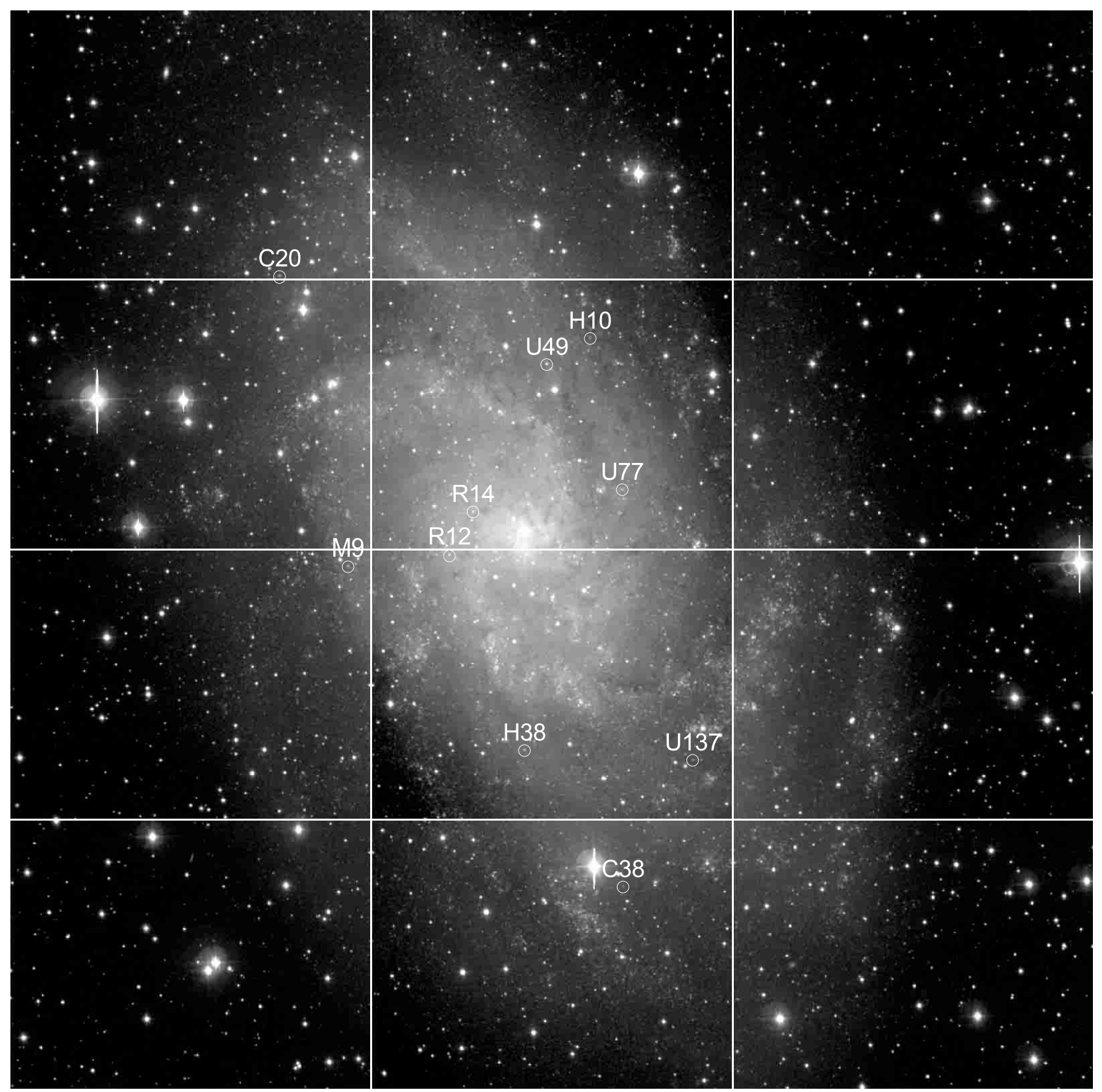

Fig. 1. The image of M 33 in filter BATC07 (5785 $\AA$ ) and the positions of the sample globular clusters. The center of the image is located at $\mathrm{RA}=01^{\mathrm{h}} 33^{\mathrm{m}} 50^{\mathrm{s}} 58 \mathrm{Dec}=30^{\circ} 39^{\prime} 08^{\prime \prime} .4(\mathrm{~J} 2000.0)$. North is up and east is to the left.

Schmidt Telescope of Beijing Astronomical Observatory (BAO), located at the Xinglong station. A Ford Aerospace $2048 \times 2048$ CCD camera with $15 \mu \mathrm{m}$ pixel size is mounted at the Schmidt focus of the telescope. The field of view of the CCD is $58^{\prime} \times 58^{\prime}$ with a pixel scale of $1^{\prime \prime} .7$. The multicolour BATC filter system includes 15 intermediate-band filters, covering the total optical wavelength range from 3000 to $10000 \AA$ (see Fan et al. 1996). The images of M 33 covering the whole optical body of M33 were accumulated in 13 intermediate band filters with a total exposure time of about 32.75 hours from September 23, 1995 to August 28, 2000. The CCD images are centered at RA $=01^{\mathrm{h}} 33^{\mathrm{m}} 50.58$ and Dec $=30^{\circ} 39^{\prime} 08^{\prime \prime} .4(\mathrm{~J} 2000)$. The dome flat-field images were taken by using a diffuse plate in front of the correcting plate of the Schmidt telescope. For flux calibration, the Oke-Gunn primary flux standard stars HD 19445, HD 84937, BD + 262606 and BD + 174708 were observed during photometric nights. The parameters of the filters and the statistics of the observations are given in Table 1. Figure 1 is the image of M 33 in filter BATC07 (5785 $\AA$ ), the circles in which indicate the positions of the sample clusters in this paper.

\subsection{Image data reduction}

The data were reduced with standard procedures, including bias subtraction and flat-fielding of the CCD 
Table 1. Parameters of the BATC filters and statistics of observations.

\begin{tabular}{cccccc}
\hline \hline No. & Name & cw $^{\mathrm{a}}(\AA)$ & Exp. (hr) & N.img $^{\mathrm{b}}$ & $\mathrm{rms}^{\mathrm{c}}$ \\
\hline 1 & BATC03 & 4210 & $00: 55$ & 04 & 0.024 \\
2 & BATC04 & 4546 & $01: 05$ & 04 & 0.023 \\
3 & BATC05 & 4872 & $03: 55$ & 19 & 0.017 \\
4 & BATC06 & 5250 & $03: 19$ & 15 & 0.006 \\
5 & BATC07 & 5785 & $04: 38$ & 17 & 0.011 \\
6 & BATC08 & 6075 & $01: 26$ & 08 & 0.016 \\
7 & BATC09 & 6710 & $01: 09$ & 08 & 0.006 \\
8 & BATC10 & 7010 & $01: 41$ & 08 & 0.005 \\
9 & BATC11 & 7530 & $02: 07$ & 10 & 0.017 \\
10 & BATC12 & 8000 & $03: 00$ & 11 & 0.003 \\
11 & BATC13 & 8510 & $03: 15$ & 11 & 0.005 \\
12 & BATC14 & 9170 & $01: 15$ & 05 & 0.011 \\
13 & BATC15 & 9720 & $05: 00$ & 26 & 0.009 \\
\hline
\end{tabular}

a Central wavelength for each BATC filter.

b Image numbers for each BATC filter.

${ }^{\mathrm{c}}$ Zero point error, in magnitude, for each filter as obtained from the standard stars.

images, with an automatic data reduction software named PIPELINE I developed for the BATC multi-colour sky survey (Fan et al. 1996; Zheng et al. 1999). The flatfielded images of each colour were combined by integer pixel shifting. The cosmic rays and bad pixels were corrected by comparison of multiple images during combination. The images were re-centered and position calibrated using the HST Guide Star Catalog. The absolute flux of intermediate-band filter images was calibrated using observations of standard stars. Fluxes as observed through the BATC filters for the Oke-Gunn stars were derived by convolving the SEDs of these stars with the measured BATC filter transmission functions (Fan et al. 1996). Column 6 in Table 1 gives the zero point error, in magnitude, for the standard stars in each filter. The formal errors we obtain for these stars in the 13 BATC filters are $\$ 0.02 \mathrm{mag}$. This indicates that we can define the standard BATC system to an accuracy of $\lesssim 0.02 \mathrm{mag}$.

\subsection{Integrated photometry}

For each globular cluster, the PHOT routine in DAOPHOT (Stetson 1987) was used to obtain magnitudes. In order to avoid contamination from nearby objects, a smaller aperture of 6.1 , which corresponds to a diameter of 4 pixels in Ford CCDs, was adopted. Considering the large seeing of the Xinglong station (the typical seeing is $2^{\prime \prime}$ in the Xinglong station), aperture corrections were computed using isolated stars. Finally, the SEDs for ten globular clusters were obtained. Table 2 contains the following information: Col. 1 is cluster name which is taken from Christian \& Schommer (1982). Column 2 to Col. 14 show the magnitudes of different bands. Second line of each star cluster is the uncertainties of magnitude of corresponding band. The uncertainties given for the magnitudes of each filter are obtained from DAOPHOT's PHOT routine. For each cluster, the background sky level was determined in an annulus of $\sim 14^{\prime \prime}-22^{\prime \prime}$.

\subsection{Comparison with previous photometry}

Using the Landolt standards, Zhou et al. (2002) presented the relationships between the BATC intermediate-band system and $U B V R I$ broadband system by the catalogs of Landolt $(1983,1992)$ and Galadí-Enríquez et al. (2000). We show the coefficients of the fits in Eqs. (1) and (2).

$$
\begin{aligned}
m_{B}= & m_{04}+(0.2218 \pm 0.033)\left(m_{03}-m_{05}\right) \\
& +0.0741 \pm 0.033 \\
m_{V}= & m_{07}+(0.3233 \pm 0.019)\left(m_{06}-m_{08}\right) \\
& +0.0590 \pm 0.010 .
\end{aligned}
$$

By Eqs. (1) and (2), we transform the magnitudes of ten halo globular clusters in BATC03, BATC04 and BATC05 bands to ones in $B$ band, and in BATC06, BATC07 and BATC0 8 bands to ones in $V$ band. Christian \& Schommer (1982) (hereafter CS1982) also obtained photometry for these clusters. Figure 2 plots the comparison of $V$ (BATC) and $(B-V)$ (BATC) photometry with previously published measurements (Christian \& Schommer 1982), and Table 3 shows this comparison. The mean $V$ magnitude and colour differences (this paper's values minus the values of Christian \& Schommer 1982) are $\langle\Delta V\rangle=$ $-0.006 \pm 0.017$ and $\langle\Delta(B-V)\rangle=-0.125 \pm 0.040$, respectively. The mean $V$ magnitude difference is very small, but the mean colour difference is somewhat large. Christian \& Schommer (1982) presented BVR photometry for 60 star clusters in M 33 using the KPNO video camera mounted on the $2.1 \mathrm{~m}$ telescope. They used NGC 2264 NGC 2419, NGC 7006, and M15 for flux calibration. Christian \& Schommer (1988) presented BVI photometry of 71 M 33 star clusters based on CCD frames obtained for 13 M 33 fields. They used the standard stars selected from the list of Landolt (1983) for flux calibration. By comparing the values of $B-V$ for the overlapping star clusters in Christian \& Schommer $(1982,1988)$, a large difference in the photometric measurements was found. For H38, U50, and U102, the differences of $B-V$ are larger than $0.15(0.21,0.17$, and 0.24 , respectively). The remaining overlapping nine clusters have a mean colour difference (the values of Christian \& Schommer 1988 minus the values of Christian \& Schommer 1982) $\langle\Delta(B-V)\rangle=-0.059 \pm 0.024$. Table 4 shows the comparison of $B V$ colour photometry for the overlapping star clusters in Christian \& Schommer (1982, 1988), the colour differences of which are smaller than 0.15. By analyzing the comparison above, we suggest that the offset in colour between Christian \& Schommer (1982) and this paper may be partly from the uncertainty of photometry of Christian \& Schommer (1982), since Christian \& Schommer (1982) only used NGC 2264, NGC 2419, NGC 7006, and M 15 for flux calibration. The remaining offset may come from the uncertainty of photometry in BATC03, BATC04 and BATC05 filters, since there is about an uncertainty of 
Table 2. SEDs of ten halo globular clusters.

\begin{tabular}{cccccccccccccc}
\hline $\begin{array}{c}\text { Cluster } \\
(1)\end{array}$ & 03 & 04 & 05 & 06 & 07 & 08 & 09 & 10 & 11 & 12 & 13 & 14 & 15 \\
$(2)$ & $(3)$ & $(4)$ & $(5)$ & $(6)$ & $(7)$ & $(8)$ & $(9)$ & $(10)$ & $(11)$ & $(12)$ & $(13)$ & $(14)$ \\
\hline U49 & 16.99 & 16.56 & 16.46 & 16.27 & 16.06 & 15.98 & 15.82 & 15.78 & 15.70 & 15.58 & 15.52 & 15.48 & 15.40 \\
& 0.022 & 0.018 & 0.014 & 0.015 & 0.010 & 0.011 & 0.011 & 0.010 & 0.010 & 0.009 & 0.010 & 0.012 & 0.012 \\
R12 & 17.34 & 16.81 & 16.62 & 16.43 & 16.15 & 16.11 & 15.90 & 15.83 & 15.77 & 15.68 & 15.56 & 15.51 & 15.44 \\
& 0.049 & 0.036 & 0.027 & 0.029 & 0.020 & 0.021 & 0.022 & 0.023 & 0.022 & 0.019 & 0.021 & 0.020 & 0.024 \\
R14 & 17.55 & 17.04 & 16.84 & 16.59 & 16.21 & 16.14 & 15.91 & 15.81 & 15.66 & 15.55 & 15.43 & 15.32 & 15.20 \\
& 0.052 & 0.037 & 0.027 & 0.028 & 0.019 & 0.021 & 0.017 & 0.018 & 0.020 & 0.016 & 0.017 & 0.017 & 0.021 \\
M9 & 17.82 & 17.48 & 17.38 & 17.17 & 16.96 & 16.90 & 16.73 & 16.68 & 16.60 & 16.53 & 16.45 & 16.39 & 16.38 \\
& 0.046 & 0.034 & 0.025 & 0.026 & 0.016 & 0.018 & 0.016 & 0.018 & 0.018 & 0.017 & 0.025 & 0.021 & 0.026 \\
U77 & 17.94 & 17.51 & 17.38 & 17.20 & 17.05 & 16.97 & 16.77 & 16.75 & 16.68 & 16.59 & 16.58 & 16.49 & 16.41 \\
& 0.066 & 0.047 & 0.042 & 0.039 & 0.025 & 0.024 & 0.033 & 0.024 & 0.030 & 0.028 & 0.030 & 0.031 & 0.044 \\
H38 & 18.03 & 17.68 & 17.52 & 17.27 & 17.06 & 16.99 & 16.80 & 16.71 & 16.69 & 16.61 & 16.55 & 16.46 & 16.49 \\
& 0.035 & 0.028 & 0.021 & 0.020 & 0.016 & 0.016 & 0.015 & 0.015 & 0.016 & 0.015 & 0.022 & 0.019 & 0.026 \\
C20 & 18.44 & 18.05 & 17.87 & 17.70 & 17.50 & 17.42 & 17.24 & 17.26 & 17.13 & 17.00 & 16.90 & 16.84 & 16.81 \\
& 0.037 & 0.026 & 0.022 & 0.022 & 0.017 & 0.020 & 0.017 & 0.020 & 0.019 & 0.019 & 0.025 & 0.021 & 0.039 \\
C38 & 18.71 & 18.35 & 18.28 & 18.05 & 17.98 & 17.88 & 17.78 & 17.70 & 17.73 & 17.73 & 17.80 & 17.80 & 17.72 \\
& 0.080 & 0.050 & 0.040 & 0.058 & 0.029 & 0.040 & 0.032 & 0.037 & 0.034 & 0.041 & 0.058 & 0.061 & 0.089 \\
H10 & 19.27 & 18.73 & 18.54 & 18.21 & 17.96 & 17.76 & 17.53 & 17.51 & 17.31 & 17.21 & 17.19 & 17.10 & 17.03 \\
& 0.093 & 0.055 & 0.043 & 0.038 & 0.030 & 0.030 & 0.033 & 0.031 & 0.029 & 0.029 & 0.033 & 0.036 & 0.053 \\
U137 & 19.21 & 18.88 & 18.68 & 18.42 & 18.22 & 18.10 & 17.88 & 17.80 & 17.81 & 17.74 & 17.71 & 17.54 & 17.61 \\
& 0.082 & 0.058 & 0.043 & 0.043 & 0.030 & 0.030 & 0.029 & 0.031 & 0.033 & 0.036 & 0.049 & 0.042 & 0.065 \\
\hline
\end{tabular}

Table 3. Comparison of cluster photometry with previous measurements and the data of adopted reddening values.

\begin{tabular}{cccccccc}
\hline \hline $\begin{array}{c}\text { Cluster } \\
(1)\end{array}$ & $\begin{array}{c}\text { RA (J2000) } \\
(2)\end{array}$ & $\begin{array}{c}\text { Dec (J2000) } \\
(3)\end{array}$ & $\begin{array}{c}V \text { (CS1982) } \\
(4)\end{array}$ & $\begin{array}{c}V \text { (BATC) } \\
(5)\end{array}$ & $\begin{array}{c}B-V(\text { CS1982) } \\
(6)\end{array}$ & $\begin{array}{c}B-V(\text { BATC }) \\
(7)\end{array}$ & $\begin{array}{c}E(V-I) \\
(8)\end{array}$ \\
\hline U49 & $01: 33: 45.04$ & $30: 47: 46.7$ & 16.25 & $16.21 \pm 0.018$ & 0.68 & $0.55 \pm 0.032$ & $0.07 \pm 0.02$ \\
R12 & $01: 34: 07.98$ & $30: 38: 38.0$ & $\ldots$ & $16.31 \pm 0.036$ & 077 & $0.74 \pm 0.064$ & $0.05 \pm 0.03$ \\
R14 & $01: 34: 02.45$ & $30: 40: 39.3$ & $\ldots$ & $16.41 \pm 0.035$ & 0.68 & $0.86 \pm 0.065$ & $\ldots$ \\
M9 & $01: 34: 30.17$ & $30: 38: 12.8$ & 17.06 & $17.11 \pm 0.030$ & 0.72 & $0.54 \pm 0.058$ & $0.04 \pm 0.03$ \\
U77 & $01: 33: 28.68$ & $30: 41: 35.2$ & 17.19 & $17.18 \pm 0.045$ & 0.67 & $0.53 \pm 0.084$ & $0.08 \pm 0.03$ \\
H38 & $01: 33: 52.13$ & $30: 29: 03.7$ & 17.24 & $17.21 \pm 0.028$ & 0.83 & $0.67 \pm 0.049$ & $0.04 \pm 0.01$ \\
C20 & $01: 34: 44.21$ & $30: 52: 18.8$ & 17.67 & $17.65 \pm 0.031$ & 0.77 & $0.60 \pm 0.050$ & $0.03 \pm 0.03$ \\
C38 & $01: 33: 30.66$ & $30: 22: 21.6$ & 18.10 & $18.10 \pm 0.061$ & 0.73 & $0.42 \pm 0.098$ & $0.04 \pm 0.02$ \\
H10 & $01: 33: 35.12$ & $30: 49: 00.0$ & 18.23 & $18.16 \pm 0.052$ & 0.96 & $0.80 \pm 0.100$ & $0.25 \pm 0.03$ \\
U137 & $01: 33: 14.28$ & $30: 28: 22.9$ & 18.30 & $18.38 \pm 0.054$ & 0.83 & $0.70 \pm 0.101$ & $0.09 \pm 0.03$ \\
\hline
\end{tabular}

Table 4. Comparison of CCD photometry (CS88) with photographic material (CS82).

\begin{tabular}{ccc}
\hline \hline $\begin{array}{c}\text { Cluster } \\
(1)\end{array}$ & $\begin{array}{c}B-V(\mathrm{CS} 88) \\
(2)\end{array}$ & $\begin{array}{c}B-V(\mathrm{CS} 82) \\
(3)\end{array}$ \\
\hline $\mathrm{C} 15$ & 0.46 & 0.53 \\
$\mathrm{C} 16$ & 0.39 & 0.49 \\
$\mathrm{C} 18$ & 0.80 & 0.73 \\
$\mathrm{C} 27$ & 0.35 & 0.37 \\
$\mathrm{C} 31$ & 0.49 & 0.66 \\
$\mathrm{U} 49$ & 0.69 & 0.68 \\
$\mathrm{U} 83$ & 0.37 & 0.49 \\
$\mathrm{U} 101$ & 0.35 & 0.42 \\
M9 & 0.66 & 0.72 \\
\hline
\end{tabular}

$0.02 \mathrm{mag}$ in the photometric measurements of each filter of these three ones (see in Table 1).

\section{Databases of simple stellar populations}

A simple stellar population (SSP) is defined to be a single generation of coeval stars, which have fixed parameters such as metallicity, initial mass function, etc. (Buzzoni 1997). SSPs are the basic building blocks of synthetic spectra of galaxies that can be used to infer the formation and subsequent evolution of the parent galaxies. They are modeled by a collection of stellar evolutionary tracks with different masses and initial chemical compositions, supplemented with a library of stellar spectra for stars at different evolutionary stages in evolution synthesis models (Jablonka et al. 1996). In order to study the integrated properties of halo globular clusters in M33, we use the SSPs of Galaxy Isochrone Synthesis Spectra Evolution Library (Bruzual \& Charlot 1996 hereafter GSSP). The main reason is that the SSPs are simple and reasonably well understood. 

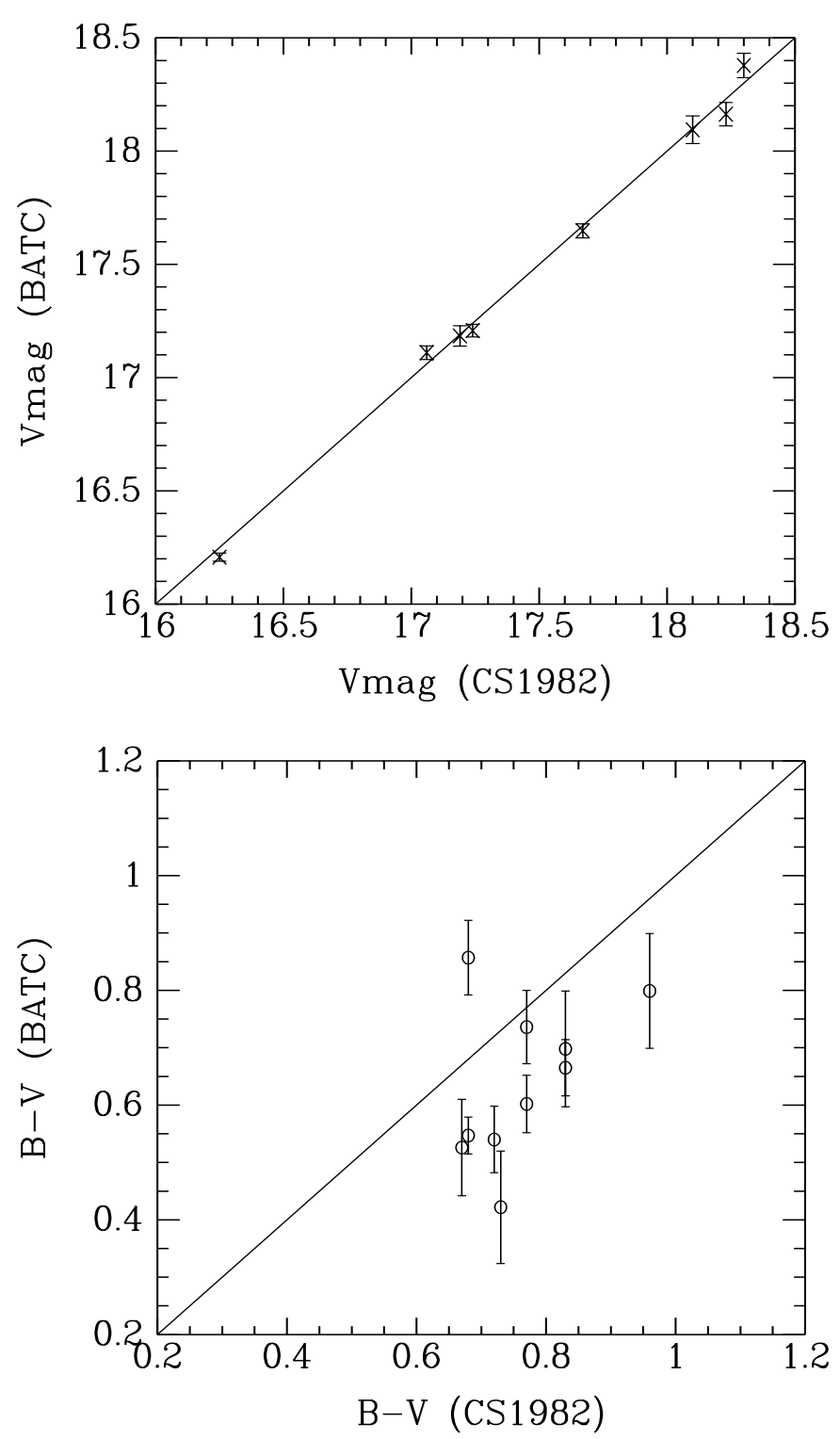

Fig. 2. Comparison of cluster photometry with previous measurements.

\subsection{Spectral energy distribution of GSSPs}

Bruzual \& Charlot (1993) presented "isochrone synthesis" as a natural and reliable approach to model the evolution of stellar populations in star clusters and galaxies. With this isochrone synthesis algorithm, Bruzual \& Charlot (1993) computed the spectral energy distributions of stellar populations with solar metallicity. Bruzual \& Charlot (1996) improved the Bruzual \& Charlot (1993) evolutionary population synthesis models. The updated version provides the evolution of the spectrophotometric properties for a wide range of stellar metallicity. They are based on the stellar evolution tracks computed by Bressan et al. (1993), Fagotto et al. (1994), and by Girardi et al. (1996), who use the radiative opacities of Iglesias et al. (1992). This library includes tracks for stars with metallicities $Z=0.0004,0.004,0.008,0.02,0.05$, and 0.1 , with the helium abundance given by $Y=2.5 Z+0.23$ (the reference solar metallicity is $\left.Z_{\odot}=0.02\right)$. The details can be seen from Ma et al. (2001).

\subsection{Integrated colours of GSSPS}

Kong et al. (2000) have obtained the age, metallicity, and interstellar-medium reddening distribution for M 81. They found the best match between the intrinsic colours and the predictions of GSSP for each cell of M 81. To determine the distributions of metallicity and age of the star clusters in M 33, we follow the method of Kong et al. (2000). Since the observational data are integrated luminosity, we need to convolve the SED of GSSP with BATC filter profiles to obtain the optical and near-infrared integrated luminosity for comparisons (Kong et al. 2000). The integrated luminosity $L_{\lambda_{i}}(t, Z)$ of the $i$ th BATC filter can be calculated with

$L_{\lambda_{i}}(t, Z)=\frac{\int F_{\lambda}(t, Z) \varphi_{i}(\lambda) \mathrm{d} \lambda}{\int \varphi_{i}(\lambda) \mathrm{d} \lambda}$,

where $F_{\lambda}(t, Z)$ is the spectral energy distribution of the GSSP of metallicity $Z$ at age $t, \varphi_{i}(\lambda)$ is the response functions of the $i$ th filter of the BATC filter system $(i=3,4, \ldots, 15)$, respectively.

The absolute luminosity can be obtained if we know the distance to M33 and the extinction along the line of sight. However, we do not know these parameters exactly. So, we should work with the colours because of their independence of the distance. We calculate the integrated colours of a GSSP relative to the BATC filter BATC08 $(\lambda=6075 \AA)$ :

$C_{\lambda_{i}}(t, Z)=L_{\lambda_{i}}(t, Z) / L_{6075}(t, Z)$.

As a result, we obtain intermediate-band colours for 6 metallicities from $Z=0.0004$ to $Z=0.1$.

\section{Reddening correction, metallicity and age of halo globular cluster}

In general, the SED of a stellar system depends on age, metallicity and reddening along the line of sight. The effects of age, metallicity and reddening are difficult to separate (e.g., Calzetti 1997; Origlia et al. 1999; Vazdekis et al. 1997). Older age, higher metallicity or larger reddening all lead to a redder SEDs of stellar systems in the optical (Mollà et al. 1997; Bressan et al. 1996). In order to obtain intrinsic colours for these star clusters, we must correct for reddening.

\subsection{Reddening correction}

The observed colours are affected by two sources of reddening: (1) the foreground extinction in our Milky Way and (2) internal reddening due to varying optical paths through the disk of the parent galaxy. Sarajedini (1994) presented the relation between the metal abundance and reddening of a globular cluster, by which the two parameters can be simultaneously derived using the shape of 
Table 5. Metallicity and age distribution of ten halo globular clusters.

\begin{tabular}{|c|c|c|c|c|c|c|c|c|c|c|}
\hline Cluste & $\begin{array}{r}\text { Age }[\log \mathrm{yr}] \\
\quad Z=0.00\end{array}$ & $R^{2}$ & $\begin{array}{r}\text { Age }[\log \mathrm{yr}] \\
Z=0.00\end{array}$ & 04 & $\begin{array}{l}\text { Age }[\log \mathrm{yr}] \\
\quad Z=0.0\end{array}$ & $R^{2}$ & $\begin{array}{r}\text { Age }[\log \mathrm{yr}] \\
Z=0.0\end{array}$ & $R^{2}$ & $\begin{array}{l}\text { Model of metallicity } \\
\text { adopted } Z([\mathrm{Fe} / \mathrm{H}])\end{array}$ & $\begin{array}{l}\text { Age [log yr] } \\
\text { adopted }\end{array}$ \\
\hline $\mathrm{U} 49$ & $10.30 \pm 0.14$ & 2 & $060+014$ & S & 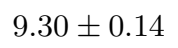 & ${ }^{0}+2>$ & $t^{2}+2$ & & & 4 \\
\hline $\mathrm{R} 12$ & & 0.014 & .18 & 0.001 & 8 & 0.002 & 18 & 0. & & .18 \\
\hline M9 & & 0.002 & & 0.0 & & & & 0.0 & & \\
\hline U77 & & 0.002 & 11 & 0.001 & 9.20 & 0.001 & 9.01 & 0.003 & & 11 \\
\hline H38 & 1 & 0.002 & 7 & 0.001 & 7 & 0.0 & 9.16 & 0.0 & & \\
\hline $\mathrm{C} 20$ & 11 & 0.012 & 11 & 0.0 & 11 & 0.004 & 9.2 & 0.006 & & .11 \\
\hline C38 & $9.28 \pm 0.02$ & 0.006 & 8.96 & 0.0 & 2 & 0.0 & 8.81 & 0.020 & & .02 \\
\hline H10 & $10.30 \pm$ & 0.009 & $9.90=$ & 0.002 & $9.51=$ & 0.003 & $9.26 \pm$ & 0.007 & ) & .14 \\
\hline U137 & $10.27 \pm 0.05$ & 0.003 & $9.57 \pm 0.05$ & 0.004 & $9.28 \pm 0.05$ & 0.005 & $9.16 \pm 0.05$ & 0.009 & $0.0004(-1.70)$ & $10.27 \pm 0.05$ \\
\hline
\end{tabular}

Table 6. Metallicity, age and reddening for R14.

\begin{tabular}{|c|c|c|c|c|c|c|c|c|c|c|c|c|c|}
\hline $\begin{array}{r}\text { Age }[\log \mathrm{yr}] \\
Z=0\end{array}$ & $\begin{array}{l}B- \\
004\end{array}$ & $\overline{R^{2}}$ & $\overline{\mathrm{Ag}}$ & $\begin{array}{c}E \\
Z=\end{array}$ & $\begin{array}{l}-V \\
.004\end{array}$ & $\overline{R^{2}}$ & ge & & $\begin{array}{l}-V) \\
.02\end{array}$ & & $\begin{array}{l}\text { Model of metallicity } \\
\text { adopted } Z([\mathrm{Fe} / \mathrm{H}])\end{array}$ & $\begin{array}{l}\text { Age [log yr] } \\
\text { adopted }\end{array}$ & $\begin{array}{c}E(B-V) \\
\text { adopted }\end{array}$ \\
\hline $10.30 \pm 0.10$ & 0.35 & 0.004 & 9.11 & 010 & 0.38 & 0.003 & 9.23 & & 020 & 0.0 & $0.004(-0.70)$ & 0.10 & 0.38 \\
\hline
\end{tabular}

the red giant branch (RGB), the $V$ magnitude of the horizontal branch (HB), and the apparent $V-I$ colour of the RGB at the level of the HB. Using this relation, Sarajedini et al. (1998) obtained the reddening (including both the foreground extinction in our Milky Way and internal reddening) for nine clusters except for R14. For $\mathrm{R} 14$, we may determine its reddening by fitting the intrinsic colours and integrated colours of GSSP (see Sect. 4.2). The reddening is an important parameter in our paper, but it is difficult to determine. As a first step, we only adopted the values presented by Sarajedini et al. (1998). They are included in Table 3 . We converted the values of $E(V-I)$ to $E(B-V)$ using a combination of the relation $(E(V-I) / E(B-V)=1.35)$ published by Zinn \& Barnes (1996). Besides, we adopted the extinction curve presented by Zombeck (1990).

\subsection{Metallicity and age distribution}

Since we model the stellar populations by SSPs, the intrinsic colours for each star cluster are determined by two parameters: age, and metallicity. In this section, we will determine these two parameters for these star clusters simultaneously by a least square method. The best fit age and metallicity are found by minimizing the difference between the intrinsic colours and integrated colours of GSSP:

$R^{2}(n, t, Z)=\sum_{i=3}^{15}\left[C_{\lambda_{i}}^{\mathrm{intr}}(n)-C_{\lambda_{i}}^{\mathrm{ssp}}(t, Z)\right]^{2}$

where $C_{\lambda_{i}}^{\mathrm{ssp}}(t, Z)$ represents the integrated colour in the $i$ th filter of a SSP with age $t$ and metallicity $Z$, and $C_{\lambda_{i}}^{\text {intr }}(n)$ is the intrinsic integrated colour for $n$th star cluster.
Multi-colour photometry of each object was compared with models for six different values of $Z$ to determine ages for globular clusters. In Table 5 (the results of R14 are listed in Table 6), ages from each model are given separately. (We only list the models of four different values of $Z$.) We also present the parameter $R^{2}$ of Eq. (5) in Tables 5 and 6 . The age of each cluster from the smallest $R^{2}$ is adopted, since this is the best overall fit to each globular cluster. Figure 3 shows a mosaic of the fit of the integrated colour of a SSP $(Z=0.0004,0.004,0.008$, and 0.02$)$ with the intrinsic integrated colour for these ten globular clusters. In Fig. 3, the thick line represents the best fit of the integrated colour of a SSP of GSSP, and filled circle represents the intrinsic integrated colour of a star cluster. From Tables 5 and 6 , we can see that eight halo globular clusters perhaps have "intermediate" ages, i.e. between 1 and 8 Gyrs. The results also show that nine of the ten halo globular clusters are metal poor. Clusters C38 and U137 are as metal poor as $[\mathrm{Fe} / \mathrm{H}]=-1.70$, $\mathrm{U} 77$ is as metal rich as $[\mathrm{Fe} / \mathrm{H}]=0.0$, respectively. The other clusters are as metal poor as $[\mathrm{Fe} / \mathrm{H}]=-0.70$. In this paper, we may estimate ages of clusters that have "intermediate" ages, however, cannot determine metallicities of clusters since the models of GSSP (Bruzual \& Charlot 1996) are not suited for a metallicity determination. Although we presented the metallicity of each cluster in Tables 5 and 6 , we only mean that, in this model of metallicity, the intrinsic integrated colour of a cluster can do the best fit with the integrated colour of a SSP at some age. The results in this study may reflect possible non-astrophysical solutions. Deep colour-magnitude diagrams would be necessary for conclusive ages.

Since the value of reddening for globular cluster R14 is not presented by other authors, we attempt to determine 


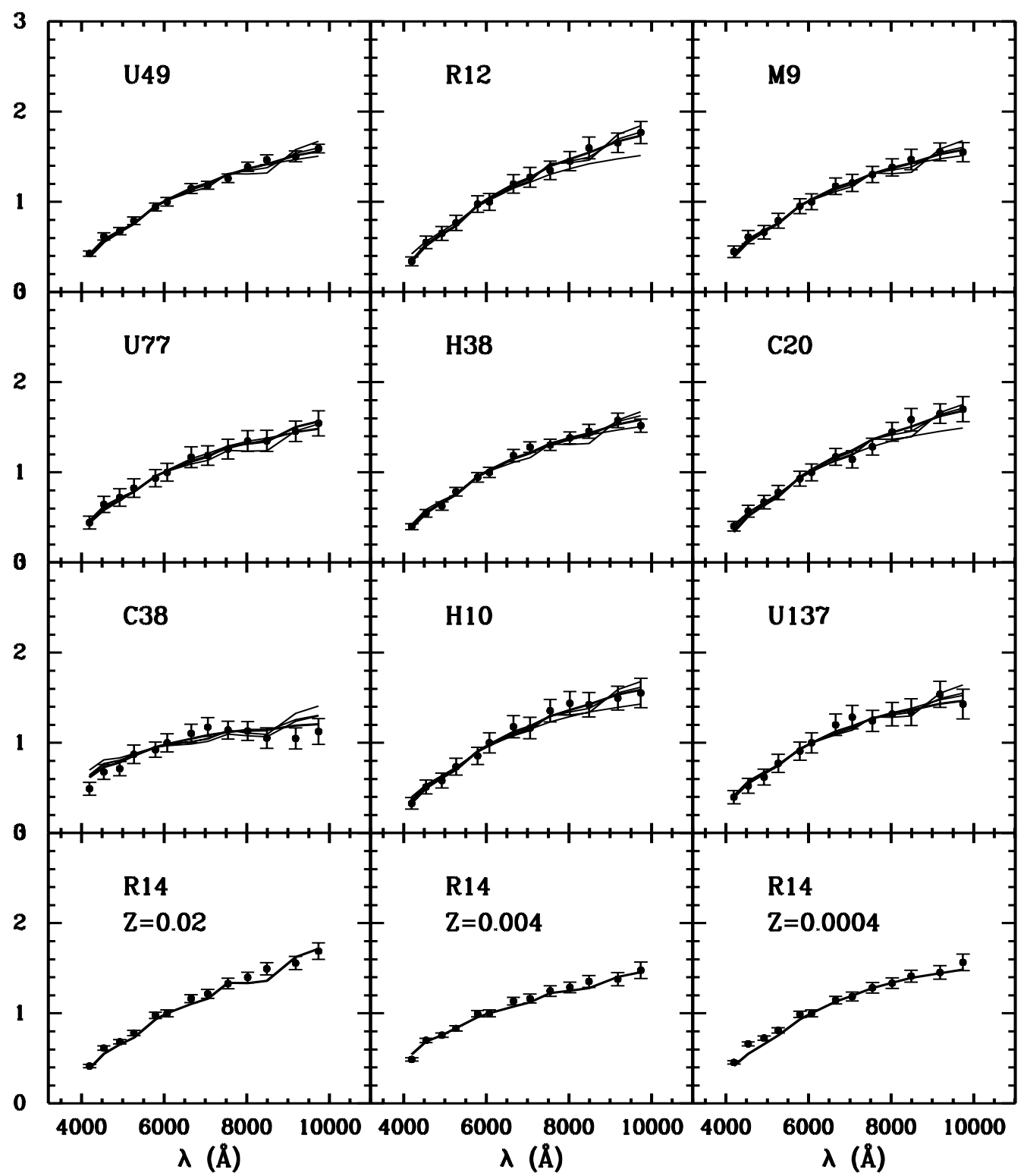

Fig. 3. Mosaic of the fit of the integrated colour of a $\operatorname{SSP}(Z=0.0004,0.004,0.008$, and 0.02) with intrinsic integrated colour for sample halo globular clusters except for R14, the intrinsic integrated colour of which is fitted by three models of metallicity $(Z=0.02,0.004$, and 0.0004). Thick line represents the best fit of the integrated colour of a SSP, and filled circle represents the intrinsic integrated colour of a star cluster.

it and age simultaneously by fitting the intrinsic colours and integrated colours of GSSP using Eq. (5). We select three models of metallicity $(Z=0.02,0.004$, and 0.0004$)$. The results are listed in Table 6 .

\section{Summary}

In this paper, we have, for the first time, obtained the SEDs of ten halo globular clusters of M 33 in 13 intermediate colours with the BAO 60/90 cm Schmidt telescope. Below, we summarize our main conclusions.

1. Using the images obtained with the Beijing Astronomical Observatory 60/90 cm Schmidt Telescope in 13 intermediate-band filters from 3800 to $10000 \AA$, we obtained SEDs of ten halo globular clusters, that were detected by Christian \& Schommer (1982), and corrected their coordinates using the HST Guide Star Catalog.

2. Using theoretical stellar population synthesis models, we obtained the distributions of age for ten clusters. The results only suggest that eight of the ten halo globular clusters have "intermediate" ages, i.e. between 1 and 8 Gyrs, however, these results may reflect possible nonastrophysical solutions.

Acknowledgements. We would like to thank two anonymous referee for providing valuable comments in the scientific contents and language of this paper. We are greatly indebted to Dr. Habing for a very careful reading of the manuscript and comments which improved the presentation of our results. We are grateful to the Padova group for providing us with a set of theoretical isochrones and SSPs. We also thank G. Bruzual and 
S. Charlot for sending us their latest calculations of SSPs and for explanations of their code. The BATC Survey is supported by the Chinese Academy of Sciences, the Chinese National Natural Science Foundation and the Chinese State Committee of Sciences and Technology. The project is also supported in part by the National Science Foundation (grant INT 93-01805) and by Arizona State University, the University of Arizona and Western Connecticut State University.

\section{References}

Bressan, A., Fagotto, F., Bertelli, G., \& Chiosi, C. 1993, A\&AS, 100, 647

Bressan, A., Chiosi, C., \& Tantalo, R. 1996, A\&A, 311, 425

Bruzual, G., \& Charlot, S. 1993, ApJ, 405, 538

Bruzual, G., \& Charlot, S. 1996, Documentation for GISSEL96 Spectral Synthesis Code.

Buzzoni, A. 1997, in Cosmological Parameters and Evolution of the Universe, ed. K. Sato, IAU Symp., 183, 18

Calzetti, D. 1997, AJ, 113, 162

Christian, C. A., \& Schommer, R. A. 1982, ApJS, 49, 405

Christian, C. A., \& Schommer, R. A. 1988, AJ, 95, 704

Fagotto, F., Bressan, A., Bertelli, G., \& Chiosi, C. 1994, A\&AS, 105, 39

Fan, X. H., Burstein, D., Chen, J.-S., et al. 1996, AJ, 112, 628

Galadí-Enríquez, D., Trullols, E., \& Jordi, C. 2000, A\&AS, 146,169
Girardi, L., Bressan, A., Chiosi, C., Bertelli, G., \& Nasi, E. 1996, A\&AS, 117, 113

Iglesias, C. A., Rogers, F. J., \& Wilson, B. G. 1992, ApJ, 397, 717

Jablonka, P., Bica, E., Pelat, D., \& Alloin, D. 1996, A\&A, 307, 385

Kong, X., Zhou, X., Chen, J., et al. 2000, AJ, 119, 2745

Landolt, A. U. 1983, AJ, 88, 439

Landolt, A. U. 1992, AJ, 104, 340

Ma, J., Zhou, X., Kong, X., et al. 2001, AJ, 122, 1796

Mollà, M., Ferrini, F., \& Diaz, A. I. 1997, ApJ, 475, 519

Origlia, L., Goldader, J. D., Leitherer, C., Schaerer, D., \& Oliva, E. 1999, ApJ, 514, 96

Sarajedini, A. 1994, AJ, 107, 618

Sarajedini, A., Geisler, D., Harding, P., \& Schommer, R. 1998, ApJ, 508, L37

Schommer, R. A., Christian, C. A., Caldwell, N., Bothun, G. D., \& Huchra, J. 1991, AJ, 101, 873

Stetson, P. B. 1987, PASP, 99, 191

Vazdekis, A., Peletier, R. F., Beckman, J. E., \& Casuso, E. 1997, ApJS, 111, 203

Zheng, Z., Shang, Z., Su, H., et al. 1999, AJ, 117, 2757

Zhou, X., et al. 2002, in preparation

Zinn, R. J., \& Barnes, S. 1996, AJ, 112, 1054

Zombeck, M. V. 1990, Handbook of Space Astronomy and Astrophysics-2nd ed. (Cambridge University Press), 104 